\title{
An XMM-Newton view of the cluster of galaxies Abell 85
}

\author{
F. Durret ${ }^{1}$, G. B. Lima Neto ${ }^{2}$, and W. Forman ${ }^{3}$ \\ 1 Institut d'Astrophysique de Paris, CNRS, 98bis Bd Arago, 75014 Paris, France \\ e-mail: durret@iap.fr \\ 2 Instituto de Astronomia, Geofísica e C. Atmosf./USP, R. do Matão 1226, 05508-090 São Paulo/SP, Brazil \\ ${ }^{3}$ Harvard Smithsonian Center for Astrophysics, 60 Garden St, Cambridge MA 02138, USA
}

Received 15 July 2004 / Accepted 17 November 2004

\begin{abstract}
We have observed the cluster of galaxies Abell 85 with XMM-Newton. These data have allowed us in a previous paper to confirm the existence of the extended $4 \mathrm{Mpc}$ filament detected by the ROSAT PSPC in the neighbourhood of this cluster, and to determine an X-ray temperature of about $\sim 2 \mathrm{keV}$. We now present a thorough analysis of the properties of the X-ray gas in the cluster itself, including temperature and metallicity maps for the entire cluster. These results show that Abell 85 had intense merging activity in the past and is not fully relaxed, even in the central region. We have also determined the individual abundances for some iron-group metals and $\alpha$-elements in various regions; the ratios of these metallicities to the iron abundance show that both supernova types Ia and II must be involved in the intra-cluster gas enrichment. Spectral analysis of the central region suggests a different redshift of the X-ray emitting gas compared to the mean cluster velocity derived from galaxy member redshifts. We discuss the implications of the difference between the $\mathrm{cD}$ galaxy redshift, the mean galaxy redshift and the hot gas redshift, as well as the possibility of several groups being accreted on to Abell 85 . Finally, we obtain the dynamical mass profile and baryon fraction taking into account the newly determined temperature profile. The dynamical mass in Abell 85 has a steep density profile, similar to the ones found in $N$-body simulations.
\end{abstract}

Key words. plasmas - galaxies: intergalactic medium - X-rays: galaxies: clusters - galaxies: clusters: individual: A85

\section{Introduction}

Clusters of galaxies are the largest and latest bound, relaxed structures to form in the Universe. They are used to probe large scale structure and evolution of galaxies in dense environments. A deep understanding of clusters is therefore necessary if they are to be effectively used as an astrophysical tool and laboratory, and detailed multi-wavelength observations of nearby clusters are one of the best means to reach a better knowledge of clusters.

The complex of clusters Abell 85/87/89 is a very wellstudied structure at both X-ray (with ROSAT, Pislar et al. 1997; Lima Neto et al. 1997; and with BeppoSAX, Lima Neto et al. 2001), and optical wavelengths, with extensive redshift and imaging catalogues (Durret et al. 1998a; Slezak et al. 1998); the mean galaxy redshift of Abell 85 is $z=0.0555$ (Durret et al. 1998b) ${ }^{1}$. As shown by Durret et al. (1998b), galaxies in the region of Abell 87 have redshifts comparable to those in Abell 85, but Abell 87 is not detected in X-rays (as confirmed by the present XMM-Newton data), suggesting that it is not a separate cluster or group. Abell 89 comprises two background structures and is not clearly detected in X-rays either, so we will not consider these two "Abell clusters" any further.

\footnotetext{
${ }^{1}$ At $z=0.0555,1$ arcmin $=90.5 h_{50}^{-1} \mathrm{kpc}$, assuming $\Omega_{\mathrm{M}}=0.3$, $\Omega_{\Lambda}=0.7$.
}

The general picture drawn from a combined X-ray (ROSAT PSPC) and optical (imaging and large redshift catalogue) analysis (Durret et al. 1998b, and references therein), shows the existence of a main cluster, of a southern blob that is probably a group at the same redshift as the main cluster, and of an extended filament (at least $4 \mathrm{Mpc}$ long). The latter may be diffuse emission or a chain of several groups of galaxies, which seem to be falling onto the main cluster, the impact region being located in projection somewhat north of the south blob.

In order to analyse better the morphology and physical properties of the X-ray gas in various regions of the main cluster and in the filament, we have observed Abell 85 with XMM-Newton. Our first results concerning the filament were described by Lima Neto et al. (2002), Durret et al. (2003). We present here the results concerning the physical properties of the X-ray gas in the main cluster. They are complementary to those of Kempner et al. (2002), who discuss the Chandra observation of Abell 85 with emphasis on the X-ray concentration at the northernmost end of the filamentary structure: the south subcluster or south blob (the filament lies outside their field).

\section{Observations and data reduction}

Two XMM-Newton (Jansen et al. 2001) observations were performed on January 7 th, 2002. The first exposure was pointed 
between the center of Abell 85 (coincident with the cD galaxy) and the south blob; the second observation was aimed at the southern filament. Both total exposure times were $12.5 \mathrm{ks}$ using the MEDIUM optical filter in standard Full Frame mode. The basic data processing (the "pipeline" removal of bad pixels, electronic noise and correction for charge transfer losses) was done with SAS V5.3. After the paper was submitted, SAS V6.0 became available and we tested that several of our results (the temperature profile, the South blob spectral fits) remained within the error bars, thus making a new analysis unnecessary.

For the spectral analysis, we have used the observations made with the EPIC/MOS1, MOS2 and PN cameras. For the EPIC/MOS cameras, after applying the standard filtering, keeping only events with FLAG $=0$ and PATTERN $\leq 12$, we have removed the observation times with flares using the light-curve in the [10-12 keV] band using a 3-sigma clip technique. The cleaned MOS 1 and MOS2 event files have remaining exposure times of $12381 \mathrm{~s}$ and $12386 \mathrm{~s}$, respectively.

We did similarly for the PN, applying the standard filtering and keeping only events with FLAG $=0$ and PATTERN $\leq 4$. The cleaned PN event file has a useful exposure time of $9039 \mathrm{~s}$.

The redistribution and ancillary files (RMF and ARF) were created with the SAS tasks rmfgen and arfgen for each camera and each region that we analysed.

The smoothed, merged image of the four cleaned MOS observations in the $0.3-5.0 \mathrm{keV}$ energy band has already been shown by Durret et al. (2003), confirming our previous detection of the "filament" with the ROSAT PSPC.

Spectra were analyzed with XSPEC 11.3. Since the spectra were rebinned, we have used standard $\chi^{2}$ minimization. We have applied both MEKAL (Kaastra \& Mewe 1993; Liedahl et al. 1995) and APEC (Chandra X-Ray Center, ATOMDB version 1.3.1) plasma models.

The metal abundances (or metallicities) are based on the solar values given by Anders \& Grevesse (1989). We chose to use these abundances for easier comparison with previous work, even though these values may not be accurate: more recent solar abundance determinations (e.g. Grevesse \& Sauval 1998; Wilms et al. 2000) give significantly lower O and Fe abundances than Anders \& Grevesse (1989). This is important to take into account when we use "variable metallicity" models in order to fit the individual metal abundances (the VMEKAL and VAPEC plasma models).

The photoelectric absorption - mainly due to neutral hydrogen in our galaxy - was computed using the cross-sections given by Balucinska-Church \& McCammon (1992), available in XSPEC.

\section{Global properties of the X-ray gas}

\subsection{Overall morphology}

The overall XMM-Newton image of Abell 85 is displayed in Fig. 1, with the various regions indicated analyzed in detail below. The main cluster, South Blob and VSSR region are clearly visible.
A zoom of the optical SDSS 2nd Data Release ${ }^{2}$ and Chandra maps of the very central region is displayed in Fig. 2. An X-ray point source which may be an AGN is visible southeast of the cluster center. The distribution of the X-ray gas towards the west and northwest edges of the central region seems somewhat more clearcut than in the rest of the cluster centre. This suggests a cold front comparable to that previously found in other clusters, e.g., Markevitch et al. (2000). A "hole" in $\mathrm{X}$-ray emission is also visible south of the cluster center.

\subsection{Temperature and metallicity maps}

The temperature and metallicity maps are made in a grid, where each pixel is $512 \times 512 \mathrm{XMM}$ EPIC physical pixels, i.e., each cell grid is $25.6^{\prime \prime} \times 25.6^{\prime \prime}$. So, from now on " 1 pixel" is actually this large 25.6 " $\times 25.6^{\prime \prime}$ "pixel". In each pixel we try a spectral fit to determine simultaneously the temperature and metallicity.

We set a minimum count number ( $\sim 600$ counts for a temperature map, $\sim 4000$ for a metallicity map) necessary for proceeding with a spectral fit using the MEKAL plasma model.

If we do not have the minimum count number in a pixel, we try a square region of $3 \times 3$ pixels; if we still do not have the minimum number of counts, we try a $5 \times 5$ pixel region. For the metallicity map only, we went up to a $7 \times 7$ pixel region. If we did not have enough counts, the pixel is ignored and we proceed to the next neighbouring pixel. This is done for all pixels in the grid, covering most of the MOS image.

When we do have enough counts, the spectral fit is done with the hydrogen column density fixed at the local galactic value - for each pixel, we estimate $N_{\mathrm{H}}$ using the task nh from FTOOLS (which is an interpolation from the Dickey \& Lockman (1990) galactic $N_{\mathrm{H}}$ table). Note that we compute the RMF and ARF matrix for each pixel in the grid.

Since the cluster covers more or less the entire XMM-Newton field, the background was taken into account by extracting spectra (for MOS1, MOS2 and PN) from the EPIC blank sky templates described by Lumb et al. (2002) and publicly available. We have applied the same filtering procedure to the background event files and extracted the spectra in all the regions. Finally, the spectra were rebinned with the grppha task, to reach at least 20 counts per energy bin. We checked that there was no difference in the soft Galactic emission (in the [0.3-1.0] keV range) between the source and background files. We also checked that the $10-12 \mathrm{keV}$ count rates were the same in our data and in the background files.

The temperature map (Fig. 3) reveals that the gas is colder near the center, as expected from its cooling flow classification. Several hotter patches are observed on the west half of the cluster. The fact that the region located just north of the south blob is hotter agrees with the scenario in which this is the "impact" region where the groups forming the filament hit the cluster, and therefore compress and heat the intracluster gas, probably through a shock.

The very steep spectrum radio source (VSSR) (Bagchi et al. 1998; Lima Neto et al. 2001) located southwest of the center

${ }^{2}$ Sloan Didital Sky Survey: http://www. sdss.org/dr2/ 


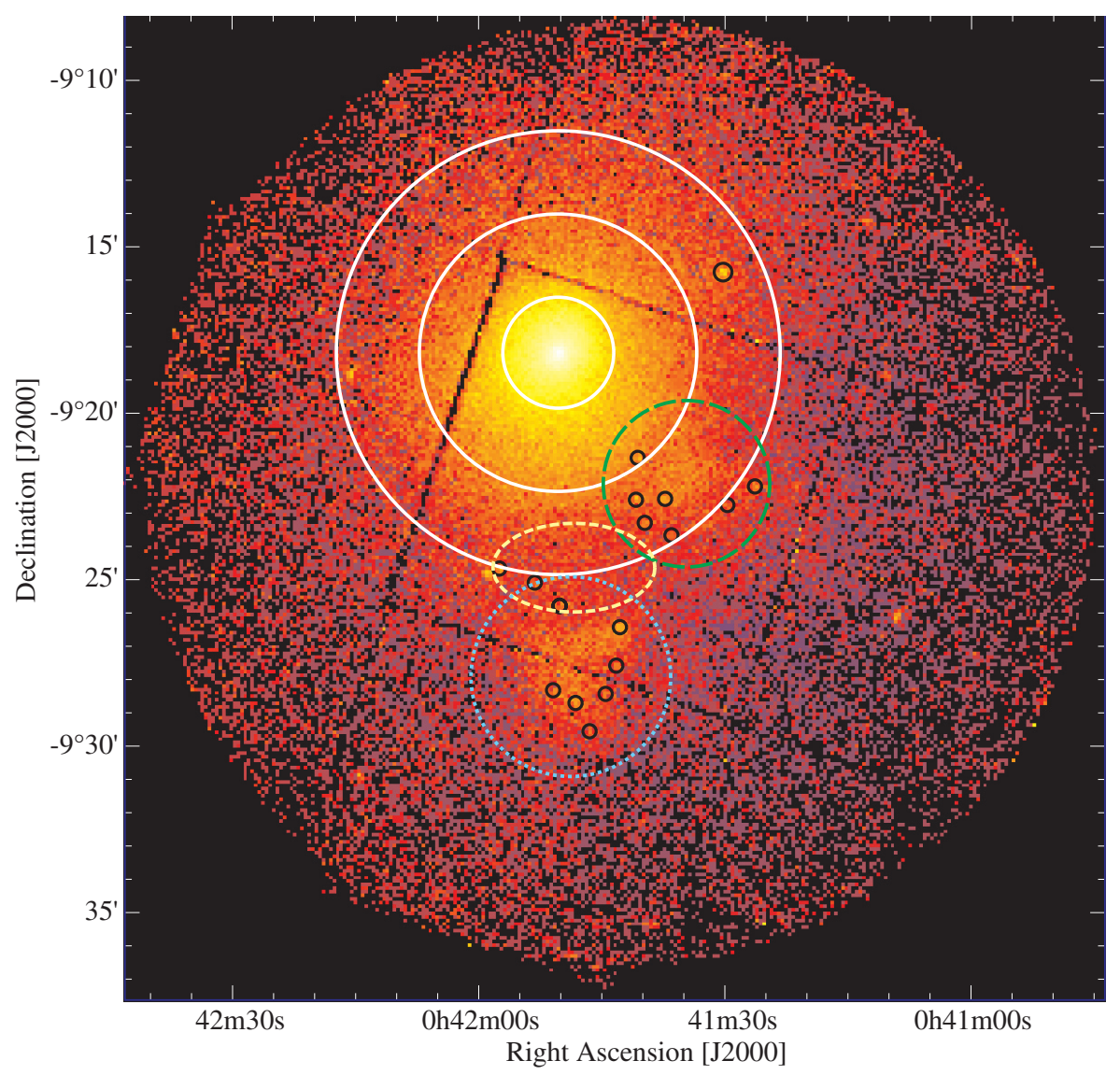

Fig. 1. XMM MOS1 and MOS2 raw image of Abell 85, with the regions analysed in detail superimposed. The concentric circles around the cluster centre (white full lines) define the centre and rings used for the detailed spectral analysis; the green dashed circle defines the VSSRS region; the yellow dashed ellipse defines the impact region, where the South Blob is probably hitting the main body of Abell 85; the blue dotted circle defines the South Blob region. The small black circles are point sources that were excluded when accumulating the spectra. The VSSRS region was also excluded from the external ring.
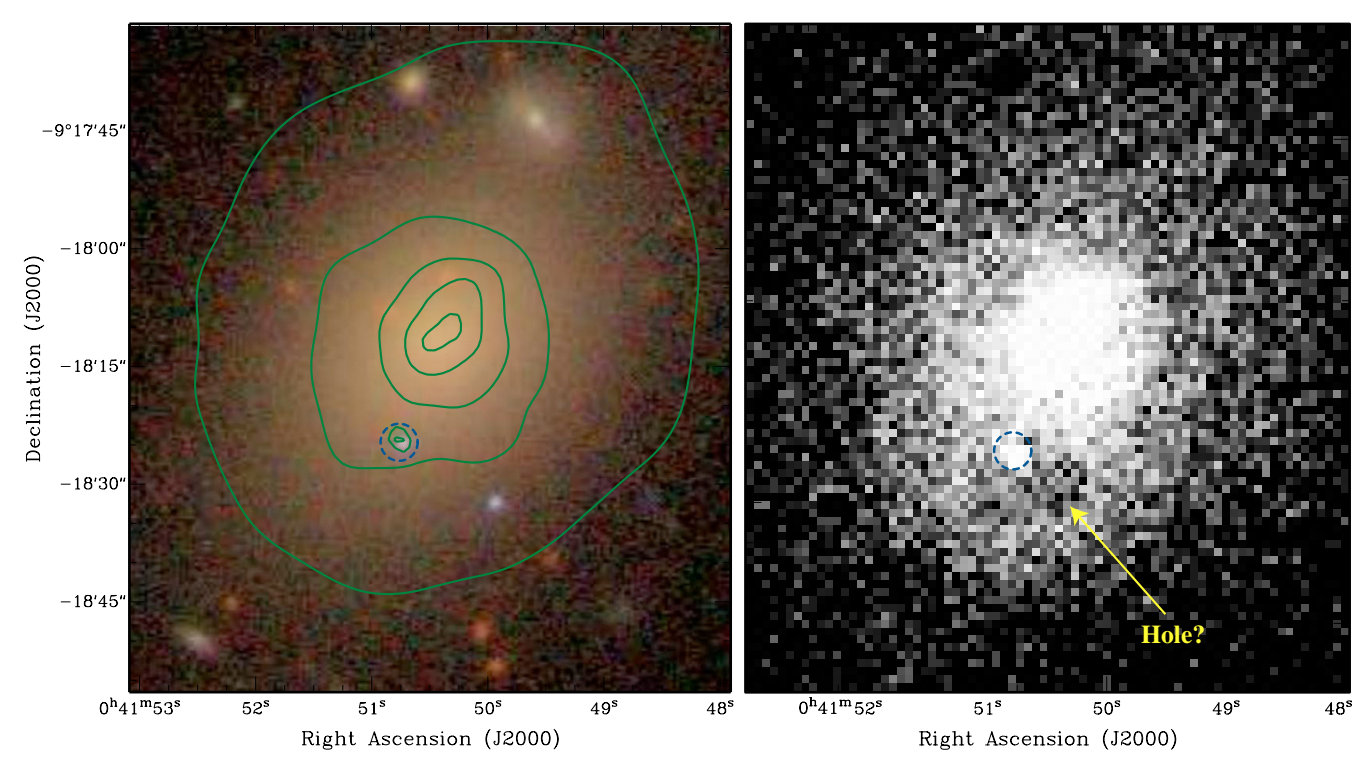

Fig. 2. Left: optical "true-color" $r-g-i$ image from the SDSS 2nd Data Release overlayed with logarithmically spaced Chandra isocontours. Right: Chandra X-ray image in the [0.3-8.0 keV] band. The small circle on both panels indicates an X-ray point source which may be an AGN. The arrow at the bottom right shows the position of a "hole" of X-ray emission south of the nucleus clearly visible on the Chandra image.

corresponds to a somewhat cooler region (see its full spectral analysis below).

Typical errors on temperatures in this map are about $10 \%$.

The metallicity map (Fig. 4) was drawn by setting the ratios of the heavy element abundances relative to that of iron equal to the solar ratios. Regions in grey are those where the spectral signal to noise ratio was not sufficient to derive a metallicity.
The central region, with an extension towards the northeast, has a notably higher metallicity, as expected from the higher density of galaxies which tend to increase the heavy element abundances in the intracluster medium through supernova winds. A higher metallicity is also observed in two zones more or less symmetrically located on either side of the central region towards the northwest and southeast, at the extremities of the 


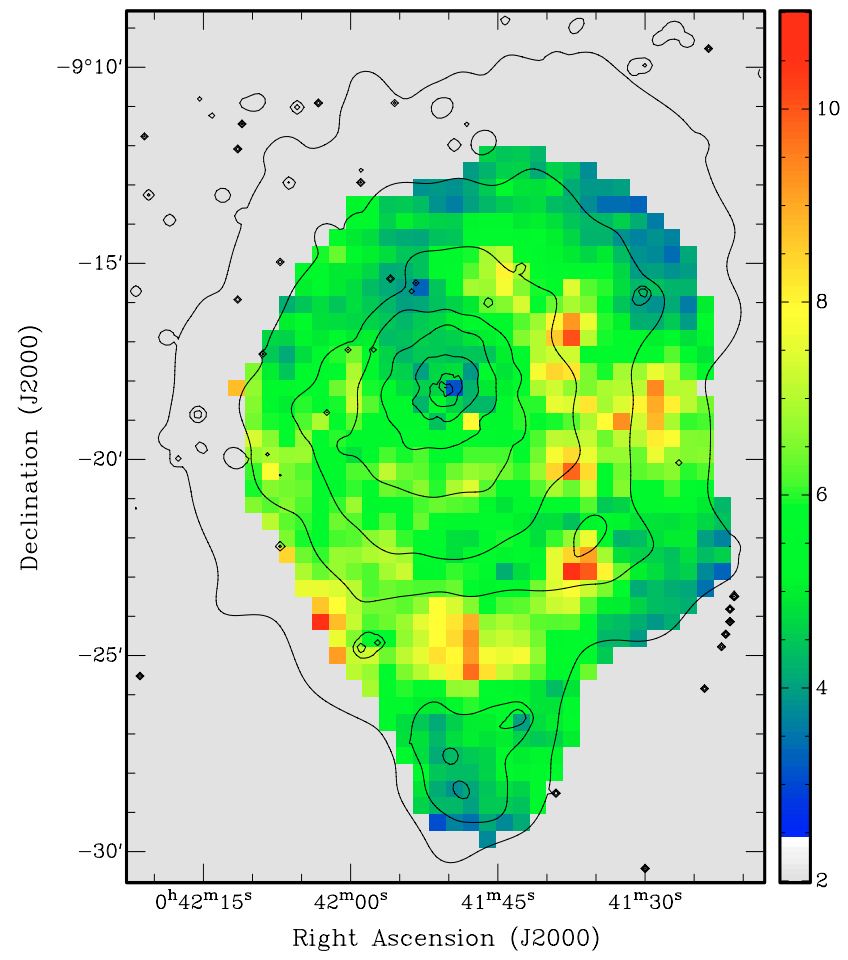

Fig. 3. Temperature map and XMM isocontours in the [0.5-8.0] keV band for Abell 85 . The scale for the temperature is $\mathrm{keV}$.

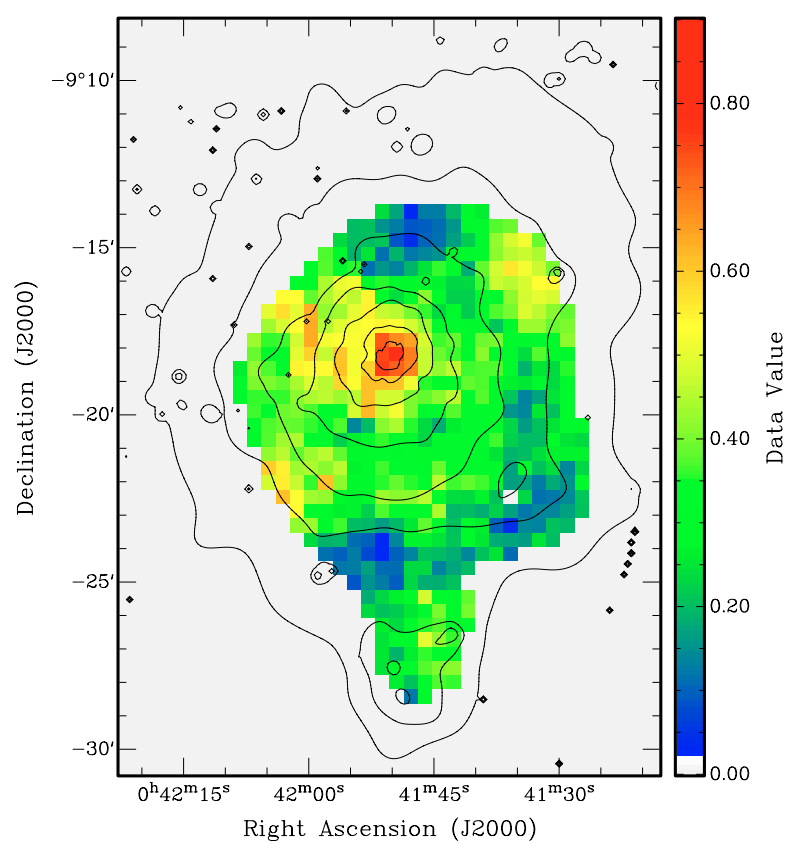

Fig. 4. Metallicity map with XMM X-ray intensity contours in the [0.5-8.0] keV band superimposed. The scale for the metallicity is in solar units.

image. The northwest region has a cooler temperature, while the southeast region has a hotter temperature, so the higher metallicities in these two zones cannot be explained by an anticorrelation between the metallicity and temperature. Typical errors on metallicities in this map are about $10-15 \%$.

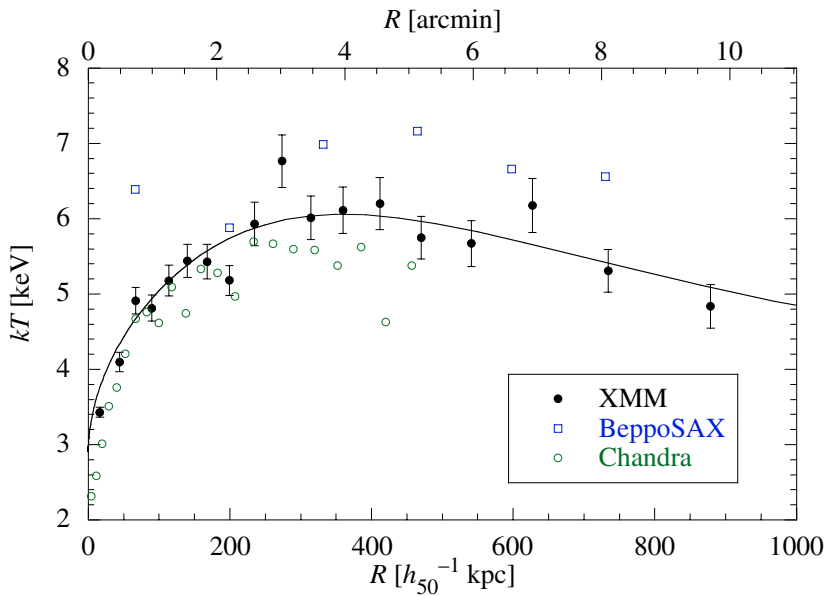

Fig. 5. Gas temperature as a function of radius obtained with XMM-Newton (filled circles), Chandra (empty circles) and BeppoSax (empty squares).

\section{Radial profiles}

We have determined the radial temperature, metallicity and absorbing hydrogen column density profiles using the combined MOS1, MOS2 and PN events. We have used circular annuli, with a width determined by requiring a minimum of about 2000 counts per annulus; this condition was obtained by trial and error, requiring a compromise between spatial resolution and accurate spectral fitting. All three parameters (temperature, metallicity and absorption) were left free to vary.

\subsection{Temperature profile}

The XMM-Newton data points for the gas temperature as a function of radius are shown together with the BeppoSax and Chandra points in Fig. 5. There is an obvious decrease of the gas temperature towards the center for radii below about $2.5 \operatorname{arcmin}(250 \mathrm{kpc})$. The temperature is constant between radii of 250 and $450 \mathrm{kpc}$, then slowly decreases outwards. Note that the overall shape of the temperature profile remains the same (within error bars) whether we exclude the south blob and VSSR regions or not.

In order to take the radial variations of the temperature into account when deriving the dynamical mass, we searched for a mathematical approximation to the temperature profile.

As a mathematical approximation to the temperature profile, we fit the following empirical law:

$T(R)=T_{0}+2 T_{0} \frac{\left(R / r_{t}\right)^{1 / 2}}{1+\left(R / r_{t}\right)^{2}}$,

where $r_{t}$ is a scale parameter and $T_{0}$ is the central temperature.

The best least-squares fit to the data is obtained for $k T_{0}=$ $2.83 \pm 0.04 \mathrm{keV}$ and $r_{t}=(630 \pm 50) h_{50}^{-1} \mathrm{kpc}$. Figure 5 shows the best fit temperature profile superposed on the data points; the agreement is fairly good.

We use the profile given by Eq. (1) as an approximation to the deprojected temperature profile, $T(r)$. For physically realistic gas models, the difference between the actual (deprojected) 


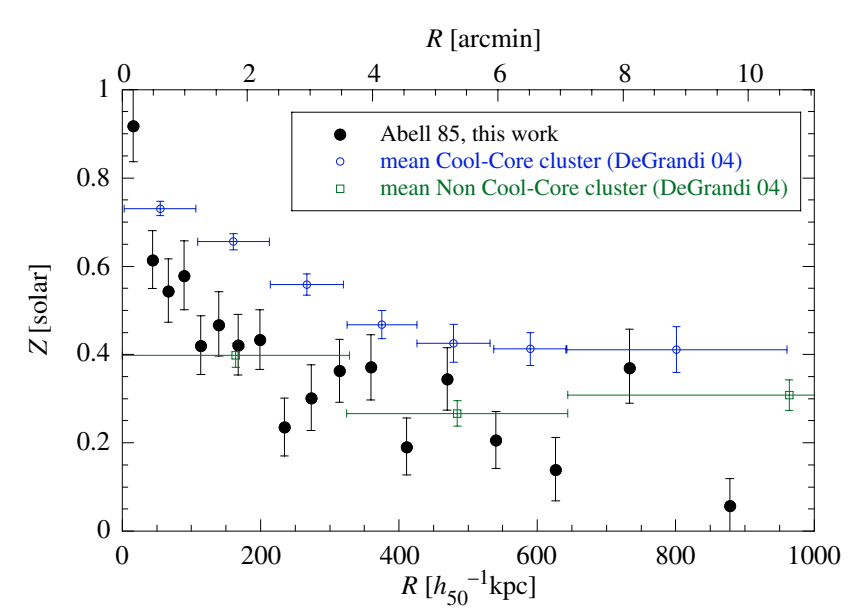

Fig. 6. Gas metallicity profile from our XMM-Newton data. For comparison we also plot the De Grandi et al. (2004) data for "cool-core" and non "cool-core" clusters.

gas temperature and the measured emission weighted temperature should be smaller than $\sim 10 \%$, well within the observational uncertainties (e.g., Markevitch et al. 1999; Komatsu \& Seljak 2001). Using Eq. (1) as an approximation to the true temperature profile allows us to compute analytically the dynamical mass profile and the corresponding total (dark matter plus baryon) density profile (cf. Sect. 6.2).

\subsection{Metallicity profile}

The radial metallicity profile is displayed in Fig. 6. The metallicity is close to solar in the innermost radial bin, and decreases to about $0.3 Z_{\odot}$ for radii larger than about $350 \mathrm{kpc}$.

The metallicity profile of Abell 85 has a shape similar to the mean metallicity profiles obtained by De Grandi et al. (2004) for their sample of "cool-core" clusters, but with systematically lower values. The metal abundance of Abell 85 is closer to the non "cool-core" values.

\subsection{Absorption profile}

The hydrogen absorption column profile is shown in Fig. 7. For comparison we also indicate the galactic value derived from Dickey \& Lockman (1990) (using the task nh from FTOOLS) and Hartmann \& Burton (1997) (using the value quoted by Hartmann \& Burton 1997). The values from Dickey \& Lockman are well above ours, while that of Hartmann \& Burton is in better agreement with ours.

Although the error bars are too large for this effect to be clearly significant, we can note that the absorption column tends to be smaller than the Galactic value at almost all radii, as previously found in a totally independent analysis based on ROSAT PSPC data (Pislar et al. 1997). Since the galactic $N_{\mathrm{H}}$ maps are made on a coarser resolution than the scale at which clusters are observed in X-rays, the actual $N_{\mathrm{H}}$ in the direction of Abell 85 may be smaller than the value quoted in these maps.

The absorption profile is apparently not flat: there may be stronger absorption in the central region, within 3 arcmin. Such a behaviour was already noticed with BeppoSAX data

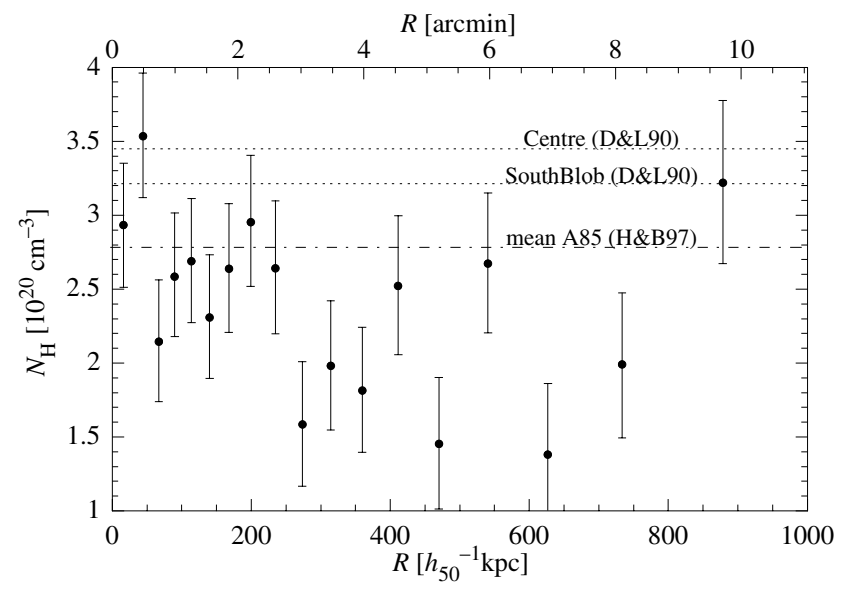

Fig. 7. Hydrogen absorption column profile from our XMM-Newton data. The two horizontal dotted lines indicate the Galactic absorption columns towards the Centre of Abell 85 and towards the South Blob obtained with the FTOOLS nh task, based on Dickey \& Lockman (1990, see text for details); the dot-dashed line is the galactic $N_{\mathrm{H}}$ value from Hartmann \& Burton (1997), cf. Arabadjis \& Bregman (1999).

by Lima Neto et al. (2001) in Abell 85, and in other clusters (e.g., Pointecouteau et al. 2004, from XMM data). The origin of such an absoption gradient, if present, is still controversial (Allen 2000; Pointecouteau et al. 2004, and references therein); a possible hypothesis is absorption due to very cool molecular gas and/or dust grains. Notice however that the error bars are still too large to confirm the absorption gradient.

\section{Physical properties and metal abundances in various regions}

We now analyze in detail the spectral properties of various regions of the cluster. A list of the X-ray point sources excluded in the various fits is given in Table 1; all the excluded zones around point sources have radii of 12 arcsec, except for source 3 which is a particularly bright Seyfert (see below). We derive the temperatures and iron abundances, and wherever possible the abundances of several other heavy elements, without fixing their ratios to the iron abundance equal to the solar ratios. An obvious requirement for this is to have enough counts in the spectra. As an example, there are 63000, 68 000, 38000 , 12300, 14800 and 5600 PN counts in the Centre, Ring 1, Ring 2, South blob, VSSR and Impact regions respectively (after excluding point sources).

\subsection{The central zone and two concentric rings}

We first analyze three regions drawn in Fig. 1, starting with a circular region of radius $R=100$ arcsec encompassing the cluster center (hereafter region "Center"). This corresponds more or less to the cooling flow zone, where the temperature drops, and therefore a constant temperature may not be a very good approximation.

We then extracted two regions corresponding to concentric annuli, where the temperature is expected to be roughly constant: one between radii of 100 and 250 arcsec (Ring 1), 
Table 1. Point sources excluded in the various spectral fits, sorted by RA.

\begin{tabular}{ccc}
\hline \hline Source number & RA $(\mathrm{J} 2000)$ & Dec $(\mathrm{J} 2000)$ \\
\hline 1 & 004126.354 & -092212.09 \\
2 & 004129.705 & -092245.70 \\
$3^{*}$ & 004130.167 & -091547.85 \\
4 & 004136.516 & -092340.12 \\
5 & 004137.273 & -092234.52 \\
6 & 004139.759 & -092317.72 \\
7 & 004140.625 & -092120.92 \\
8 & 004140.841 & -092236.12 \\
9 & 004142.810 & -092625.90 \\
10 & 004143.242 & -092735.07 \\
11 & 004144.539 & -092826.27 \\
12 & 004146.486 & -092933.47 \\
13 & 004148.216 & -092842.27 \\
14 & 004150.150 & -092547.59 \\
15 & 004150.920 & -092819.87 \\
16 & 004153.165 & -092505.71 \\
17 & 004157.490 & -092440.10 \\
\hline
\end{tabular}

* Seyfert galaxy.

the other between radii of 250 and $400 \operatorname{arcsec}$ (Ring 2). Two small circular zones were excluded from Ring 2: one of radius 24 arcsec centered on the Seyfert galaxy (source 3 in Table 1) and one of radius 80 arcsec centered on the VSSR. It was not possible to extract zones further out with a sufficient number of counts to estimate independently the various heavy element abundances.

For these three regions, we have simultaneously fit the spectra obtained with all three detectors (MOS1, MOS2 and PN) with the VAPEC and VMEKAL models (Kaastra \& Mewe 1993; Liedahl et al. 1995), using the photoelectric absorption given by Balucinska-Church \& McCammon (1992). For the central region, we also fit the spectra with a CEVMKL model, which takes into account the existence of a cooling flow. The energy ranges taken into account were $0.3-10.0 \mathrm{keV}$ for the two MOS spectra and $0.5-8.0 \mathrm{keV}$ for the PN spectrum. The Galactic absorption and redshift were left free to vary. For several elements, abundances could not be estimated, and were frozen to 0.3 times the solar value.

Figure 8 shows the spectra for regions Center, Ring 1 and Ring 2, together with the best VMEKAL or CEVMKL (coolingflow MEKAL model) fits and residuals in terms of the individual $\chi^{2}$ contribution of each energy bin.

Table 2 summarizes the results obtained for the various fits in these three regions. The iron abundance is estimated with a typical precision of $3 \%$ in the Center and better than $10 \%$ in the two Rings. The other elements measured in the central zone are: $\mathrm{O}, \mathrm{Ne}, \mathrm{Mg}, \mathrm{Si}, \mathrm{S}, \mathrm{Ca}$ and $\mathrm{Ni}$. $\mathrm{Mg}$ and $\mathrm{S}$ are not detected in the Rings.

The abundances of the various metals relative to solar are not at all the same from one element to another. In the central region, $\mathrm{Fe}$ and $\mathrm{O}$ are about 0.3 and $0.4 Z_{\odot}$ respectively, while $\mathrm{Ne}$ and $\mathrm{Si}$ are somewhat higher and $\mathrm{Ni}$ is much higher.

Clear metallicity gradients are also present, all elements but $\mathrm{Ca}$ being notably less abundant in the Rings than in the Center,

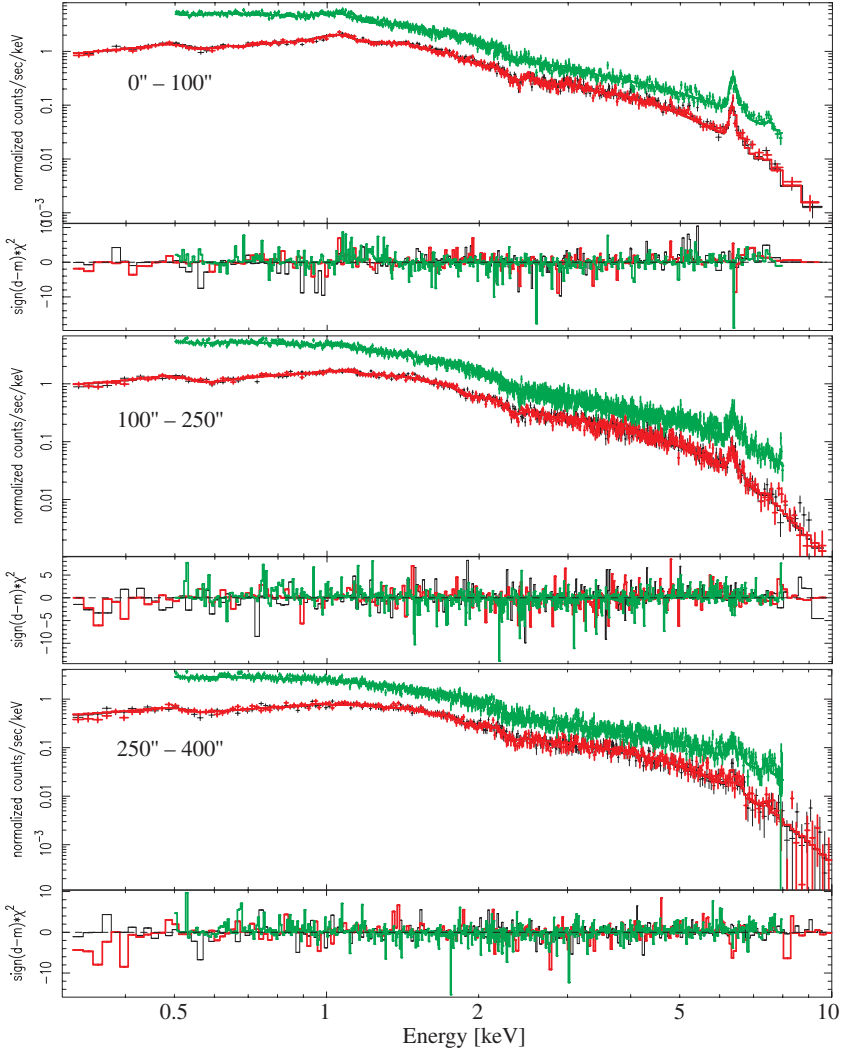

Fig. 8. Best spectral fits with residues for XMM-Newton MOS1, MOS2 and PN. Top: central region $\left(R<100^{\prime \prime}\right)$ fit with a coolingflow model (CEVMKL); middle: Ring 1 fit with a VMEKAL $\left(100^{\prime \prime}<\right.$ $\left.R<250^{\prime \prime}\right)$; bottom: Ring 2 fit with a VMEKAL $\left(250^{\prime \prime}<R<400^{\prime \prime}\right)$.

suggesting that there is little or no radial mixing or convection of the gas.

Fluxes and luminosities were calculated based on the best fit for the three detectors independently. We give in Table 4 the averages of these quantities in the [0.5-2.0 keV] and $[2.0-10.0 \mathrm{keV}]$ bands obtained from MOS1 and MOS2. The bolometric X-ray luminosities (integrated in the [0.001$100 \mathrm{keV}$ ] interval) are also given. Typical errors are of the order of $10 \%$.

Interestingly, the redshift derived from the various X-ray gas fits in the central region is between 0.0530 and 0.0535 , corresponding to velocities of $c z=15900-16050 \mathrm{~km} \mathrm{~s}^{-1}$. Figure 9 illustrates the redshift dependency on the quality of the fit. It seems clear that the X-ray emitting gas in the central 4 arcmin is best fit with $z=0.0533$.

On the other hand, the mean optical redshift of Abell 85 is 0.0555 , corresponding to a velocity of $c z=16650 \mathrm{~km} \mathrm{~s}^{-1}$ (Durret et al. 1998a). The mean optical velocity of the cluster is therefore larger than that of the X-ray gas in the innermost 100 arcsec radius region by $\sim 600 \mathrm{~km} \mathrm{~s}^{-1}$. This difference is significant only at a $2 \sigma$ level since the MOS (PN) energy calibration is accurate to $5 \mathrm{eV}(10 \mathrm{eV})$, translating to a velocity of $250 \mathrm{~km} \mathrm{~s}^{-1}\left(500 \mathrm{~km} \mathrm{~s}^{-1}\right)$. Since Abell 85 is known to show velocity substructures, we extracted from the velocity catalogue of Durret et al. (1998a) the galaxies in the innermost 100 arcsec radius region and with velocities in the cluster interval, 
Table 2. Spectral fits of the central region (radius $100 \mathrm{arcsec}$ ) with three different models, and of regions Ring 1 (radius 100 to 250 arcsec) and Ring 2 (radius 250 to 400 arcsec) with two different models. Abundances are in solar units and error bars are $1 \sigma$. Values of $N_{\mathrm{H}}$ are in units of $10^{20} \mathrm{~cm}^{-2}$.

\begin{tabular}{|c|c|c|c|c|c|c|c|}
\hline $\begin{array}{l}\text { Region } \\
\text { Model }\end{array}$ & $\begin{array}{c}\text { Center } \\
\text { vmekal }\end{array}$ & $\begin{array}{l}\text { Center } \\
\text { cevmkl }\end{array}$ & $\begin{array}{l}\text { Center } \\
\text { vapec }\end{array}$ & $\begin{array}{l}\text { Ring } 1 \\
\text { vmekal }\end{array}$ & $\begin{array}{l}\text { Ring } 1 \\
\text { vapec }\end{array}$ & $\begin{array}{l}\text { Ring } 2 \\
\text { vmekal }\end{array}$ & $\begin{array}{c}\text { Ring } 2 \\
\text { vapec }\end{array}$ \\
\hline$N_{\mathrm{H}}$ & $2.50_{-0.12}^{+0.10}$ & $2.70_{-0.02}^{+0.02}$ & $2.83_{-0.002}^{+0.001}$ & $1.78_{-0.15}^{+0.18}$ & $1.985_{-0.001}^{+0.001}$ & $1.194_{-0.002}^{+0.002}$ & $1.448_{-0.002}^{+0.001}$ \\
\hline$k T(\mathrm{keV})$ & $4.30_{-0.03}^{+0.04}$ & $5.77_{-0.15}^{+0.15}$ & $4.18_{-0.03}^{+0.03}$ & $5.70_{-0.04}^{+0.09}$ & $5.69_{-0.08}^{+0.05}$ & $6.28_{-0.13}^{+0.10}$ & $6.20_{-0.14}^{+0.08}$ \\
\hline Redshift & $0.0530_{+0.0002}^{-0.0002}$ & $0.0533_{-0.0004}^{+0.0001}$ & $0.0535_{-0.0004}^{+0.0004}$ & $0.0527_{-0.0009}^{+0.0011}$ & $0.0535_{-0.0006}^{+0.0010}$ & $0.0505_{-0.0009}^{+0.0047}$ & $0.0539_{-0.0005}^{+0.0004}$ \\
\hline$\chi^{2}$ & 1741 & 1670 & 1755 & 1770 & 1766 & 1437 & 1427 \\
\hline d.o.f. & 1491 & 1490 & 1491 & 1636 & 1635 & 1346 & 1332 \\
\hline $\mathrm{O}$ & $0.25_{+0.06}^{-0.05}$ & $0.30_{-0.05}^{+0.05}$ & $0.38_{-0.06}^{+0.09}$ & $0.022_{-0.022}^{+0.089}$ & $0.20_{-0.12}^{+0.11}$ & $0.32_{-0.15}^{+0.18}$ & $0.25_{-0.19}^{+0.24}$ \\
\hline $\mathrm{Ne}$ & $0.53_{+0.10}^{-0.09}$ & . & $0.78_{-0.10}^{+0.15}$ & $0.32_{-0.11}^{+0.12}$ & $0.44_{-0.12}^{+0.19}$ & $0.75_{-0.19}^{+0.18}$ & $1.01_{-0.23}^{+0.27}$ \\
\hline $\mathrm{Mg}$ & $\ldots$ & $0.18_{-0.10}^{+0.10}$ & $0.17_{-0.12}^{+0.13}$ & $\ldots$ & $\ldots$ & $0.29_{-0.24}^{+0.24}$ & $\ldots$ \\
\hline $\mathrm{Si}$ & $0.55_{+0.07}^{-0.06}$ & $0.60_{-0.06}^{+0.06}$ & $0.56_{-0.07}^{+0.08}$ & $0.16_{-0.09}^{+0.09}$ & $0.15_{-0.10}^{+0.10}$ & $\ldots$ & $\cdots$ \\
\hline$S$ & $0.27_{+0.09}^{-0.07}$ & $0.35_{-0.08}^{+0.08}$ & $0.28_{-0.09}^{+0.09}$ & $\ldots$ & $\ldots$ & $\ldots$ & $\ldots$ \\
\hline $\mathrm{Ca}$ & $0.35_{+0.24}^{-0.21}$ & $0.37_{-0.24}^{+0.26}$ & $0.46_{-0.23}^{+0.21}$ & $0.56_{-0.31}^{+0.31}$ & $0.57_{-0.33}^{+0.29}$ & $0.79_{-0.50}^{+0.56}$ & $1.09_{-0.58}^{+0.55}$ \\
\hline $\mathrm{Fe}$ & $0.408_{+0.018}^{-0.013}$ & $0.409_{-0.011}^{+0.012}$ & $0.451_{-0.014}^{+0.014}$ & $0.271_{-0.013}^{+0.012}$ & $0.296_{-0.015}^{+0.012}$ & $0.236_{-0.021}^{+0.021}$ & $0.261_{-0.020}^{+0.024}$ \\
\hline $\mathrm{Ni}$ & $1.39_{+0.24}^{-0.25}$ & $1.54_{-0.24}^{+0.24}$ & $1.25_{-0.29}^{+0.19}$ & $0.46_{-0.28}^{+0.27}$ & $\ldots$ & $1.34_{-0.49}^{+0.54}$ & $1.37_{-0.45}^{+0.75}$ \\
\hline
\end{tabular}

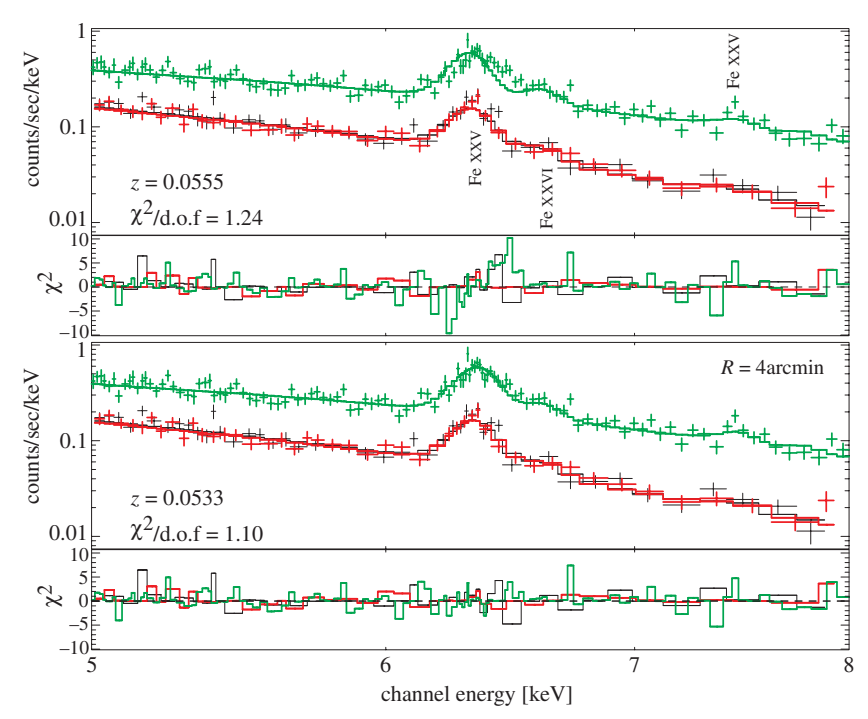

Fig. 9. Spectral MEKAL fit within the central 4 arcmin using the three XMM cameras, restricted to the $[5.0-8.0 \mathrm{keV}]$ band. Each line corresponds to one of the 3 detectors, cf. Fig. 8. Top panel: temperature and metallicity are free and the redshift is set to 0.0555 . Bottom panel, when the redshift is set free, the best fit is $z=0.0533_{-0.0009}^{+0.0015}$ ( $90 \%$ confidence level). The reduced $\chi^{2}$ is given in each panel.

as defined by Durret et al. (1998b). Four galaxies correspond to these criteria, and their mean velocity is $c z=15903 \mathrm{~km} \mathrm{~s}^{-1}$, a value consistent with the X-ray gas velocity given above.

Interestingly, the $\mathrm{cD}$ appears to have a higher velocity $c z=16734 \mathrm{~km} \mathrm{~s}^{-1}[z=0.0558 \pm 0.0002$, Durret et al. (1998a) $]$ implying that it is certainly not resting at the bottom of the cluster potential well traced by the X-ray gas. This velocity difference is in the upper range of $\mathrm{cD}$ peculiar velocities measured by Oegerle \& Hill (2001), in agreement with the general formation scenario for Abell 85 involving several mergers (e.g., Dubinski 1998).

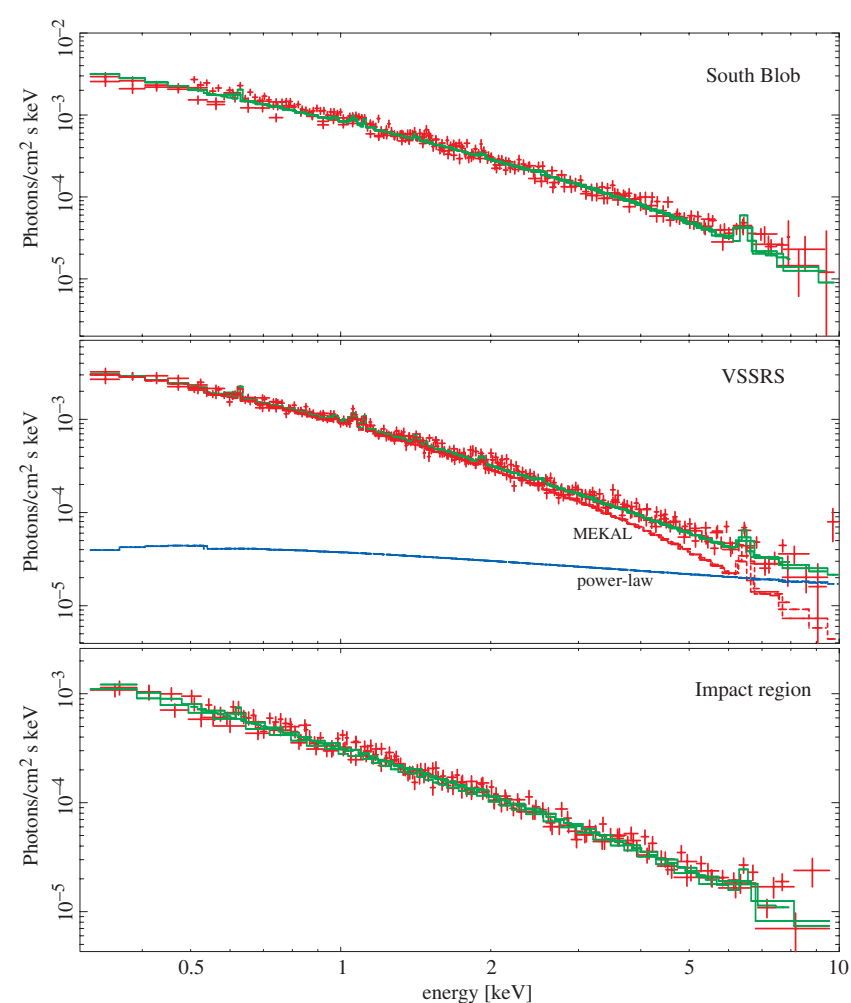

Fig. 10. Photon spectra for 3 individual regions. Top: the VSSRS region (2.5 arcmin radius region). Middle: the elliptical impact region. Bottom: the South Blob region (3.0 arcmin radius region). See text for region locations.

\subsection{The South blob}

The South blob spectrum was obtained using the Chandra image to derive the positions of the point sources, which are not all clearly visible on the XMM-Newton image, and exclude them from the XMM-Newton spectrum extracted for the South blob in a 3.0 arcmin radius region. It was then fit by MEKAL 
Table 3. Spectral fits of the South Blob (circle of radius 200 arcsec), VSSR (circle of radius 150 arcsec) and Impact regions, with two different models. Abundances are in solar units and error bars are $1 \sigma$. Values of $N_{\mathrm{H}}$ are in units of $10^{20} \mathrm{~cm}^{-2}$.

\begin{tabular}{lcccccc}
\hline \hline $\begin{array}{l}\text { Region } \\
\text { Model }\end{array}$ & $\begin{array}{c}\text { South Blob } \\
\text { vmekal }\end{array}$ & $\begin{array}{c}\text { South Blob } \\
\text { vapec }\end{array}$ & $\begin{array}{c}\text { VSSR } \\
\text { mekal }\end{array}$ & $\begin{array}{c}\text { VSSR } \\
\text { apec }\end{array}$ & $\begin{array}{c}\text { Impact } \\
\text { mekal }\end{array}$ & $\begin{array}{c}\text { Impact } \\
\text { apec }\end{array}$ \\
\hline$N_{\mathrm{H}}$ & $0.042_{-0.042}^{+0.046}$ & $0.042_{-0.041}^{+0.044}$ & $0.93_{-0.29}^{+0.30}$ & $1.00_{-0.30}^{+0.30}$ & $0.147_{-0.147}^{+0.548}$ & $0.082_{-0.082}^{+0.088}$ \\
$k T(\mathrm{keV})$ & $5.74_{-0.26}^{+0.26}$ & $5.79_{-0.27}^{+0.25}$ & $6.26_{-0.21}^{+0.22}$ & $6.23_{-0.20}^{+0.20}$ & $8.87_{-0.65}^{+0.67}$ & $8.84_{-0.60}^{+0.69}$ \\
Redshift & 0.0555 & 0.0555 & 0.0555 & 0.0555 & 0.0555 & 0.0555 \\
$\chi^{2}$ & 775 & 774 & 916 & 917 & 504 & 502 \\
d.o.f. & 758 & 758 & 882 & 882 & 533 & 533 \\
\hline $\mathrm{O}$ & $0.26_{-0.19}^{+0.24}$ & $0.40_{-0.30}^{+0.36}$ & $\ldots$ & $\ldots$ & $\ldots$ & $\ldots$ \\
$\mathrm{Ne}$ & $0.75_{-0.30}^{+0.35}$ & $1.11_{-0.43}^{+0.49}$ & $\ldots$ & $\ldots$ & $\ldots$ & $\ldots$ \\
$\mathrm{Mg}$ & $0.52_{-0.40}^{+0.46}$ & $0.68_{-0.52}^{+0.52}$ & $\ldots$ & $\ldots$ & $\ldots$ & $\ldots$ \\
$\mathrm{Si}$ & $0.25_{-0.23}^{+0.28}$ & $0.35_{-0.29}^{+0.31}$ & $\ldots$ & $\ldots$ & $\ldots$ & $\ldots$ \\
$\mathrm{Fe}$ & $0.168_{-0.031}^{+0.033}$ & $0.190_{-0.036}^{+0.037}$ & $0.201_{-0.034}^{+0.035}$ & $0.223_{-0.038}^{+0.039}$ & $0.220_{-0.084}^{+0.085}$ & $0.246_{-0.094}^{+0.095}$ \\
\hline
\end{tabular}

Table 4. X-ray fluxes and luminosities in regions Center, Ring 1 and Ring 2. Fluxes are in units of $10^{-11} \mathrm{erg} \mathrm{cm}^{-2} \mathrm{~s}^{-1}$ and luminosities in units of $10^{44} \mathrm{erg} \mathrm{s}^{-1}$.

\begin{tabular}{lcccccc}
\hline \hline Region & Center & Ring 1 & Ring 2 & South Blob & VSSR & Impact \\
\hline Flux $[0.5-2.0 \mathrm{keV}]$ & 1.35 & 1.29 & 0.65 & 0.19 & 0.22 & 0.070 \\
Flux $[2.0-10.0 \mathrm{keV}]$ & 1.81 & 2.12 & 1.14 & 0.29 & 0.38 & 0.145 \\
\hline$L_{\mathrm{X}}[0.5-2.0 \mathrm{keV}]$ & 1.88 & 1.81 & 0.91 & 0.25 & 0.31 & 0.10 \\
$L_{\mathrm{X}}[2.0-10.0 \mathrm{keV}]$ & 2.67 & 3.10 & 1.65 & 0.42 & 0.54 & 0.21 \\
$L_{\mathrm{X}, \text { bol }}$ & 6.17 & 6.64 & 3.51 & 0.92 & 1.17 & 0.44 \\
\hline
\end{tabular}

and APEC spectra. The corresponding spectrum and VMEKAL fit are shown in the top panel of Fig. 10, and results are given in Table 3. The temperature is $k T=5.75 \pm 0.25 \mathrm{keV}$. Contrary to the regions previously considered, where the redshift is left free to vary in the fit, the result here is not acceptable, in view of the mean optical redshift, which is found to correspond to a velocity of $16826 \mathrm{~km} \mathrm{~s}^{-1}$ (based on 16 galaxy redshifts). We therefore fixed the redshift to the mean cluster value of 0.0555 .

\subsection{The VSSR region}

The region called VSSR corresponds to the very steep spectrum radio source MRC 0038-096 (Large et al. 1991). Figure 11 shows the high resolution $21 \mathrm{~cm}$ VLA radio isocontours of Slee et al. (2001) superposed on the MOS1+MOS2 X-ray image.

This radio source was thought to be associated with the south-west X-ray excess already observed from ROSAT data (Lima Neto et al. 1997; Bagchi et al. 1998). Slee et al. (2001), using higher resolution radio data, also associated the radio source with the X-ray excess based on ROSAT data. With XMM data, however, the relation between the X-ray excess and the radio data is not very clear, but we can see from Fig. 11 that the high resolution VLA radio source does not coincide spatially with the X-ray excess. Such a phenomenon could happen if the relativistic electrons blow away some of the X-ray emitting gas (Boehringer et al. 1993; Fabian et al. 2000).

A spectrum was extracted in a circular region of radius 2.5 arcmin corresponding to the VSSR (see Fig. 1). The best thermal fit is obtained for $k T=6.2 \pm 0.20 \mathrm{keV}$. We also tried a double component fit, including a thermal component and a power law. The power-law component is intended to represent the inverse Compton emission of cosmic microwave background photons on the relativistic electrons responsible for the synchrotron radio emission observed in this location (Bagchi et al. 1998). However, the non-thermal component is not unambiguously disentangled from the thermal emission, and the reduced $\chi^{2}$ is not significantly better. Assuming that we have detected a non-thermal component (with the above caveat), this non-thermal X-ray flux is $1.5 \times 10^{-12} \mathrm{erg} \mathrm{s}^{-1} \mathrm{~cm}^{-2}$. The middle panel of Fig. 10 shows the best fit spectrum.

There are 8 galaxies in the VSSR region with measured redshifts, but the corresponding velocities spread between 13781 and $17800 \mathrm{~km} \mathrm{~s}^{-1}$ with no concentration at a specific velocity, so we cannot extract any kinematic information.

\subsection{The impact region}

As mentioned above, the region believed to be undergoing the impact of the infalling groups on to the main cluster is located between the south blob and the cluster center. We extracted the MOS1+MOS2+PN spectrum in an ellipse of major axis 2.43 arcmin, minor axis 1.33 arcmin and major axis position angle aligned with the east-west direction (see Fig. 1).

The spectrum of this region is shown in the bottom panel of Fig. 10 and the corresponding results for a MEKAL and an apec model are given in Table 3. As in the two previous cases, if the redshift was left free to vary in the X-ray spectral fit, we found unreasonably small values, so we had to fix the redshift to 0.0555 . As expected from the temperature map, the temperature in this zone is notably higher than in the rest of the cluster: $k T=8.8 \pm 0.6 \mathrm{keV}$, in agreement with the idea that the gas 


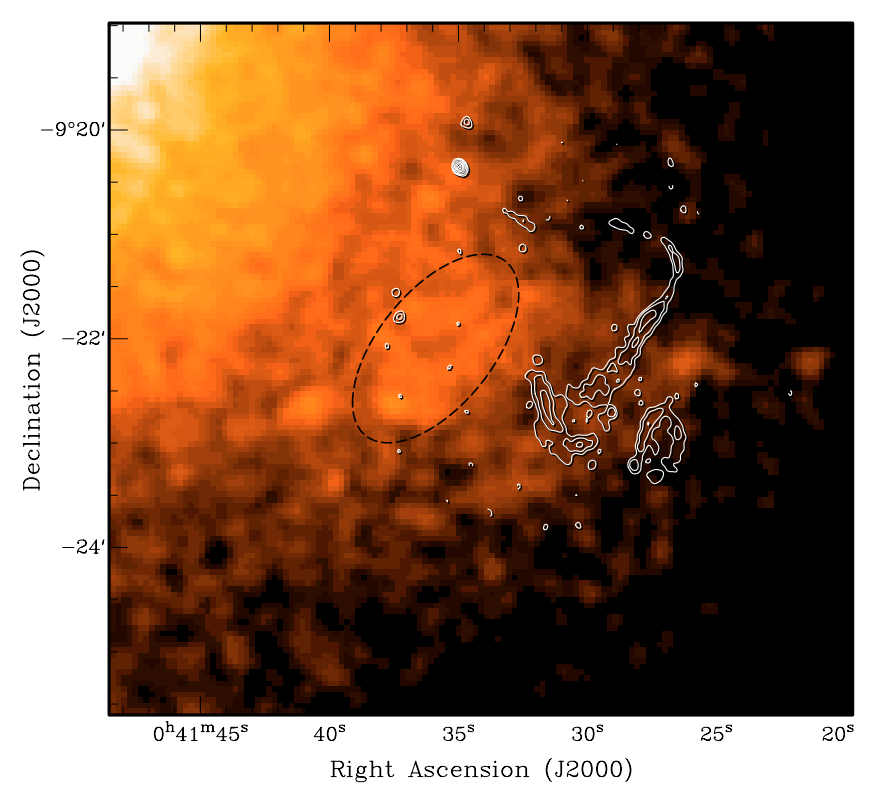

Fig. 11. MOS1+MOS2 X-ray image with the $21 \mathrm{~cm}$ radio isocontours obtained by Slee et al. (2001) overlayed. The radio source seems to be anti-correlated with the west X-ray excess emission at $\alpha \sim 0^{\mathrm{h}} 41^{\mathrm{m}} 36^{\mathrm{s}}$ and $\delta \sim-9^{\circ} 22^{\prime}$ approximately limited by the dashed ellipse.

in that region is compressed and heated by a shock due to the infall of groups on to the main cluster.

\subsection{Metallicity gradients}

The ratios of the heavy element abundances relative to iron in regions Center, Ring 1, Ring 2 and South blob are shown in Fig. 12. Various theoretical metal yield models are superimposed: "Hypernovae" (Nakamura et al. 2001), SN II, and SN Ia models WDD1, WDD2 and W7 (Nomoto et al. 1997).

In none of the four regions does any single model account for the heavy element abundances. However, Fig. 12 shows that in the Centre and Ring 1 SNIa seem capable of reproducing the observed abundances. This may be explained by the presence of a central cD galaxy which, as normal ellipticals, is deficient in type II SNe but may have a relatively high SNIa rate - roughly 16 type Ia SN per century (Cappellaro \& Turatto 2001).

On the other hand, the high $\mathrm{Ne}, \mathrm{Ca}$ and $\mathrm{Ni}$ abundances measured in Ring 2 require the contribution of SNII - this could have happened during the initial burst of star formation within the cluster elliptical galaxies (e.g., Matteucci \& Gibson 1995; Pepino et al. 2002). Note that Ring 2 is dominated by the region towards the northwest of the cluster which has a notably stronger metallicity. $\mathrm{Ne}$ and $\mathrm{Mg}$ in the South Blob are also closer to those due to SNII models while Si is consistent with SNIa models. Therefore, it does not seem possible from our data to estimate accurately the proportion of SNIa and SNII responsible for the enrichment of the intracluster medium, as in (e.g. Dupke \& White 2000a,b; Sanders et al. 2004); besides being a function of time, this proportion is probably a function of position (or local density) in the cluster (Tamura et al. 2004).

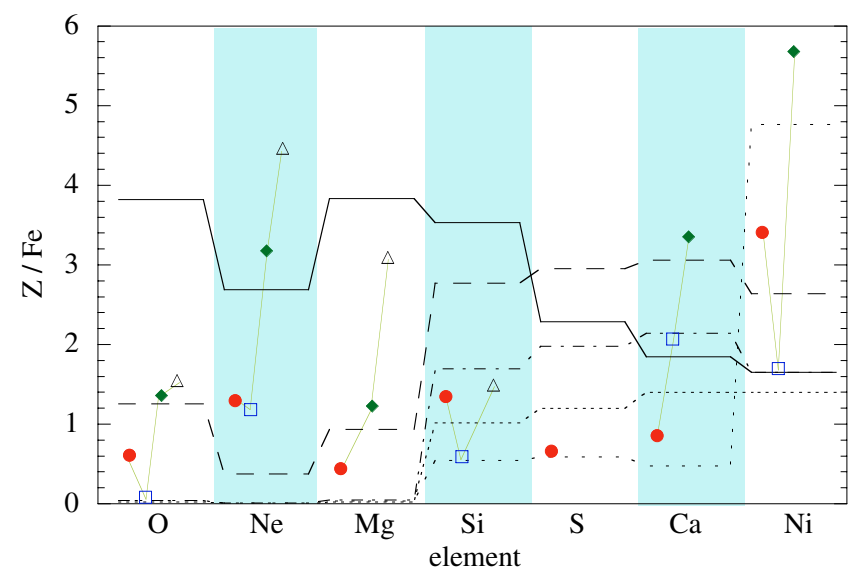

Fig. 12. Heavy element abundances relative to iron in regions Centre (filled circles), Ring 1 (empty squares), Ring 2 (filled diamonds) and South Blob (empty triangles). Various models are superimposed: SNII (full line), Hypernovae (long dashes), and SNIa WDD1 (dot-dashed), WDD2 (dotted) and W7 (spaced dots).

\section{Mass determination}

\subsection{Gas mass}

The gas density distribution is determined from the radial X-ray surface brightness profile, $\Sigma_{\mathrm{X}}$, using the fact that:

$\Sigma_{\mathrm{X}}(R) \stackrel{\propto}{\sim} \int_{R}^{\infty} n_{\mathrm{e}}^{2}(r) \frac{r \mathrm{~d} r}{\sqrt{r^{2}-R^{2}}}$

where $n_{\mathrm{e}}$ is the electron number density of the intracluster gas. For an electrically neutral gas, $n_{\mathrm{e}} \approx n_{\mathrm{H}}$ and then $\rho(r) \approx m_{\mathrm{p}} n_{\mathrm{e}}(r)$, where $m_{\mathrm{p}}$ is the proton mass.

We have used two analytical profiles to describe $\Sigma(R)$, the Sérsic (1968) law:

$\Sigma(R)=\Sigma_{0} \exp \left[-\left(\frac{R}{a}\right)^{v}\right]$,

and the $\beta$-model (Cavaliere \& Fusco-Femiano 1976):

$\Sigma(R)=\Sigma_{0}\left[1+\left(\frac{R}{R_{\mathrm{c}}}\right)^{2}\right]^{-3 \beta+1 / 2}$,

where $a$ and $R_{\mathrm{c}}$ are the scale parameters, $v$ and $\beta$ the shape parameters, and $\Sigma_{0}$ the intensity normalization.

The X-ray brightness profile of Abell 85 was obtained with the STSDAS/IRAF task ELLIPSE, using the XMM-Newton MOS1 exposure corrected [0.3-8.0 keV] band image. We have masked the CCD gaps and point sources. In Fig. 13, we show the brightness profile together with three different fits: a Sérsic profile, a $\beta$-model and a $\beta$-model excluding the four innermost points, which correspond to a known excess emission with respect to a flat core (cf. Gerbal et al. 1992; Lima Neto et al. 1997).

If all the points are included, the Sérsic law provides a better fit than the $\beta$-model; however, if the four innermost points are excluded, the Sérsic and $\beta$-models become indistinguishable.

We have chosen the Sérsic profile to describe the emissivity profile for its simplicity and capability to describe well the 


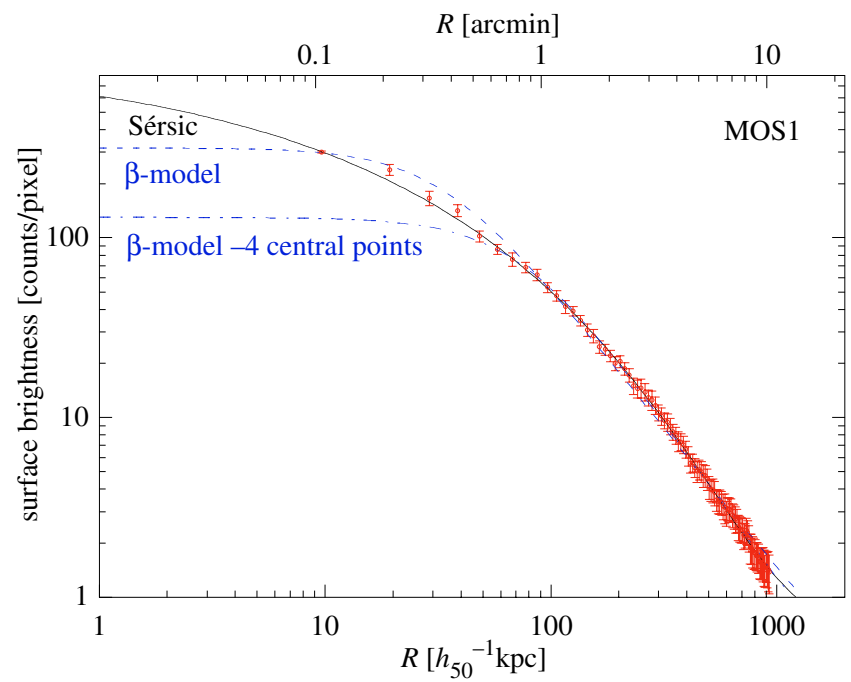

Fig. 13. Emission profile of the X-ray gas obtained from the XMM-Newton MOS1 image (the profile from MOS2 is litterally indistinguishable from MOS1); three fits are superimposed: a Sérsic law, a $\beta$-model (dashes) and a $\beta$-model excluding the four innermost points (dot-dashes).

whole profile (see e.g. Demarco et al. 2003). There is an analytical approximation to the deprojection of a Sérsic profile (Lima Neto et al. 1999). Thus, if the brightness profile is given by Eq. (3), the electron number density is:

$n_{\mathrm{e}}(r)=n_{0}(r / a)^{-p} \exp \left[-(r / a)^{v}\right]$;

$2 p=1-0.6097 v+0.05563 v^{2}$,

and, for Abell 85, a least-squares fit of the brightness profile gives $a=35 \pm 7 \mathrm{kpc}, v=0.39 \pm 0.02$ and $p=0.39 \pm 0.03$.

In order to estimate the electron density, $n_{0}$ (the intensity normalization in Eq. (5)), we integrate the bremsstrahlung emissivity along the line-of-sight within 4 arcmin in the central region and compare the result with the flux obtained by spectral fitting of the same region (the normalization parameter of the thermal spectral model in XSPEC, which is proportional to $n_{\mathrm{e}}^{2}$ ). We thus obtain $n_{0}=(77.0 \pm 6.5) \times 10^{-3} \mathrm{~cm}^{-3}$.

The gas mass as a function of radius can then simply be derived by integrating the gas density in spherical shells:

$M(<r)=\frac{4 \pi a^{3} n_{0} m_{\mathrm{p}}}{v} \gamma\left[\frac{3-p}{v},\left(\frac{r}{a}\right)^{v}\right]$,

where $\gamma(z, x) \equiv \int_{0}^{x} \mathrm{e}^{z} z^{t-1} \mathrm{~d} t$ is the incomplete gamma function. We use $\rho(r)=m_{\mathrm{p}} n_{\mathrm{e}}(r)$, supposing the gas is electronically neutral and $m_{p}$ is the proton mass. The gas mass is shown in Fig. 14.

At the limiting radius of our data, $r=10$ arcmin or about $900 h_{50}^{-1} \mathrm{kpc}$, the gas mass is $3.5 \times 10^{13} M_{\odot}$.

\subsection{Dynamical mass and baryon fraction}

In order to compute the dynamical mass, the temperature profile is introduced. Assuming hydrostatic equilibrium and

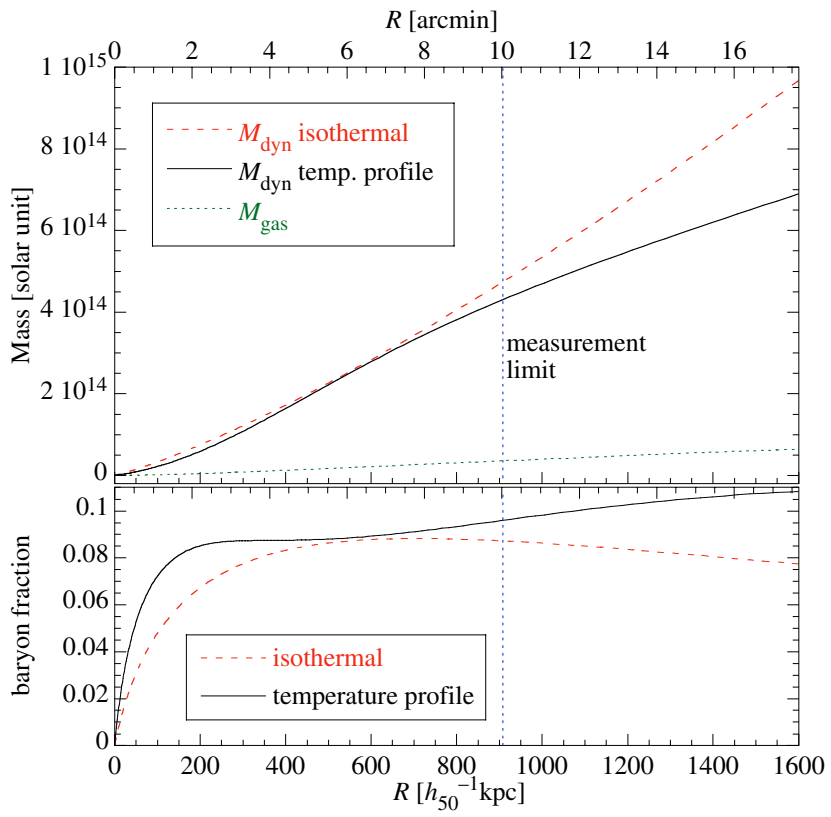

Fig. 14. Top: gas mass (dotted line) and dynamical mass (solid line when including the calculation of the temperature profile, dashed line in the isothermal case) as a function of radius. The vertical dotted line indicates the limiting radius of the data. For the isothermal case, we have adopted the mean emission temperature $k T=6.5 \mathrm{keV}$. Bottom: baryon fraction in both cases.

spherical symmetry, the Sérsic profile described above and the empirical temperature profile, Eq. (1), the dynamical mass is:

$$
\begin{aligned}
& M_{\mathrm{dyn}}(<r)=k T_{0} r \times 3.7 \times 10^{10} M_{\odot} \\
& \times \frac{\left[p+v\left(x \frac{r_{t}}{a}\right)^{v}\right]\left(1+x^{2}\right)\left(1+2 \sqrt{x}+x^{2}\right)-\sqrt{x}+3 x^{5 / 2}}{\left(1+x^{2}\right)^{2}},
\end{aligned}
$$

where $x \equiv r / r_{t}, a$ and $r_{t}$ are in $\mathrm{kpc}$ and $k T_{0}$ in $\mathrm{keV}$.

If we assume an isothermal temperature profile, the dynamical mass reduces to:

$M_{\mathrm{dyn}}^{\text {iso }}=3.7 \times 10^{10} k \operatorname{Tr}\left[p+v\left(\frac{r}{a}\right)^{v}\right] M_{\odot}$,

with $r$ and $a$ in kpc.

The dynamical mass is shown in Fig. 14 as a function of radius, both for isothermal and non-isothermal gas temperatures. For the isothermal case, we have adopted the mean emission weighted temperature $k T=6.5 \mathrm{keV}$. These two curves are very similar within the limiting radius of our data, but start to diverge further out, showing that even when the temperature does not vary very strongly with radius (see Fig. 5), it has a non negligible influence on the total mass estimate at large radius. At the last measured point, $r=10 \operatorname{arcmin}, M_{\mathrm{dyn}}=4.3 \times 10^{14} M_{\odot}$ and $M_{\text {dyn }}^{\text {iso }}=4.7 \times 10^{14} M_{\odot}$.

The dynamical and gas masses allow us to estimate the baryon fraction profile in Abell 85. The mass in baryons is computed assuming that $\sim 16 \%$ is contributed by the galaxies, i.e., $M_{\text {baryon }} \approx 1.16 M_{\text {gas }}$ (White et al. 1993).

The bottom panel in Fig. 14 shows the baryon fraction for both isothermal and non-isothermal temperature profiles. It is seen to increase strongly with radius within the inner $150 \mathrm{kpc}$, 


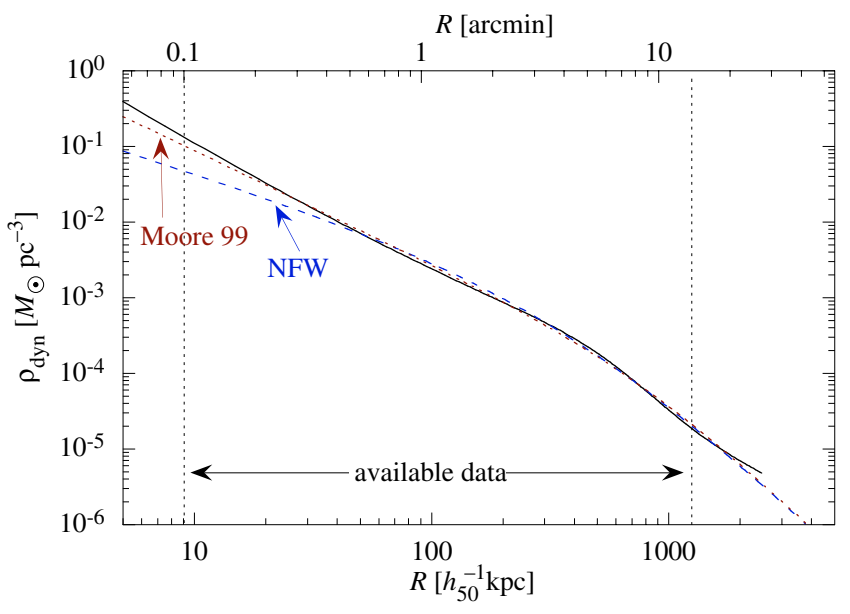

Fig. 15. Dynamical mass density as a function of radius derived from our data (full line) and compared to a NFW model (dashed line) and to a Moore99 profile (dotted line). $\rho_{\mathrm{dyn}} \propto r^{-1.9}$ is a good fit at small radii. The two vertical dotted lines indicate the spatial resolution (left) and the limit of our data (right).

then to remain roughly constant. This agrees with previous findings that dark matter is strongly peaked towards the centers of clusters (e.g. Gerbal et al. 1992; Allen et al. 2001), in agreement with numerical simulations (e.g. Rasia et al. 2004). The baryon fraction at $r=10$ arcmin is 0.09 and 0.10 for isothermal and non-isothermal profiles, respectively.

\subsection{Virial radius and halo density}

Having the dynamical mass we can compute the radius $r_{\delta}$ that corresponds to

$\overline{\rho\left(r_{\delta}\right)} / \rho_{\mathrm{c}}(z)=\delta ; \quad \overline{\rho\left(r_{\delta}\right)} \equiv \frac{M_{\mathrm{dyn}}}{4 \pi r_{\delta}^{3} / 3}$,

where $\rho_{\mathrm{c}}(z)$ is the critical density of the Universe at redshift $z$. For the SCDM and $\Lambda \mathrm{CDM}$ models, the virial radius corresponds to $\delta=180$ and 340 respectively (cf. Lacey \& Cole 1993; Bryan \& Norman 1998).

Equation (9) cannot be solved analytically when we take into account the temperature profile and the dynamical mass given by Eq. (7), but it can be solved numerically and thus we have:

$$
\begin{array}{lccccc}
\delta: & 180 & 200 & 340 & 500 & 2500 \\
r_{\delta}\left[h_{50}^{-1} \mathrm{kpc}\right]: & 2697 & 2563 & 1995 & 1671 & 789 .
\end{array}
$$

Errors on $r_{\delta}$ are about $15 \%$ ( $1 \sigma$ confidence level). Taking the virial radius as $r_{\mathrm{vir}}=2 h_{50}^{-1} \mathrm{Mpc}$, the radial range where we are able to obtain a temperature profile for Abell 85 is $0.005 \lesssim$ $r / r_{\text {vir }} \lesssim 0.45$.

We also derived the dynamical mass density as a function of radius, that can be compared to the density of dark matter halos formed in cosmological $N$-body simulations. In Fig. 15 we show the dynamical mass density compared to the Navarro et al. (1997,"NFW") "universal" profile for dark halos and to the Moore et al. (1999, "Moore99") profile.

In the interval $0.1<r<10$ arcmin, where the temperature and surface brightness are reliably determined, both theoretical profiles agree quite well (the Moore99 profile slightly better) with the density profile we obtained for Abell 85.

The characteristic radius of the NFW profile that best fits our density profile is $r_{\mathrm{NFW}}=390 h_{50}^{-1} \mathrm{kpc}$; with the above determined $r_{\mathrm{vir}}$, this implies that the concentration parameter is $c=r_{\mathrm{vir}} / r_{\mathrm{NFW}} \approx 5$. This value is in agreement with the cosmological N-body simulations of Jing \& Suto (2000), in a $\Lambda \mathrm{CDM}$ scenario.

\section{Discussion and conclusions}

Our XMM-Newton data have allowed us to obtain temperature and metallicity maps for Abell 85 and to derive heavy element abundances in various regions.

The temperatures obtained for the Center and Rings (see Table 2) agree very well with those measured by Markevitch et al. (1998) from ASCA data in his regions 1 and 6-9 respectively. On the other hand, Donnelly et al. (2003) find a fairly homogeneous temperature throughout Abell 85, except in a small zone southeast of the center, and in a large one towards the northwest edge. This difference may be due to the lower spatial resolution and sensitivity of ASCA.

The temperatures and/or metallicities of several regions seem to indicate that Abell 85 has undergone or is still undergoing merging events. However, there is no intensity enhancement whatsoever in any of the hotter or more metal rich regions, suggesting that there is no gas density discontinuity.

The $S E$ region is the only place where $k T$ and $Z$ are not anticorrelated: it is hot and metal rich; however this zone is just at the limit where metallicities can be measured, so we will not take it into account in the coming discussion.

Numerical simulations of a cluster merger such as those displayed, e.g., by Bourdin et al. (2004) in their Fig. 9, show that when two clusters merge the gas temperature is not affected immediately, but the final temperature map can look strikingly similar to the one we have derived, with various hotter zones. Different numerical simulations have also shown that star formation activity in galaxies was only briefly enhanced by a merger, for less than 3 Gyr, according to Fig. 4 in Fujita et al. (1999), but then due to ram pressure stripping galaxy star formation rates (SFR) decrease. These two sets of simulations can help us propose a possible scenario that could account for what we are seeing in Abell 85.

First, there appears to be a "filament" extending at least $4 \mathrm{Mpc}$ southeast of the cluster and probably made of groups falling on to the main cluster (Durret et al. 2003) and hitting it in the Impact region. In favour of this interpretation are two main facts: i) the temperature of the filament is about $2.0 \mathrm{keV}$, consistent with that of groups; ii) the impact region, located more or less half way between the cluster center and the south blob, is notably hotter $(8.8 \mathrm{keV})$. This is most probably the place where a particularly large group hits the main cluster.

Second, the NW region is cooler and more metal rich than the average cluster. A cooler temperature is expected in metalrich regions, but the fact that this zone is more metal rich probably implies that it has undergone star formation with a higher rate than the rest of the cluster, possibly triggered by a group merging from the NW towards the SE. Note however that 
the NW zone, as the NE one, is just at the limit where metallicities can be measured, so we should take these results with caution.

Third, the NE region has no temperature enhancement, but has a notably higher metallicity, here also suggesting metal enrichment due to enhanced star formation and/or ram pressure stripping due to a merger.

Fourth, there are hot zones distributed in an arc from south to NW. These could also be the result of a major merger coming from the NW, since as mentioned above a strong similarity is observed between our X-ray temperature map and that of the numerical simulations of a cluster merger by Bourdin et al. (2004).

If a cluster has fallen onto Abell 85 from the NW, this would be consistent with the existence of a metal-rich zone in the NW, due to a brief increase of the SFR which produced a metal enrichment of the ICM, followed by a decrease in the SFR, in agreement with the homogeneity of the metallicity map in the rest of the cluster. Such a scenario suggests that this merger has taken place more than 4 Gyr ago in order for the SFR to be presently reduced (Fujita et al. 1999). If we follow the same reasoning to interpret the NE zone, where the gas has a high metallicity but a temperature similar to the average cluster temperature, this would require a second merger coming from the NE, but notably more recently (less than $3 \mathrm{Gyr}$ ), so that star formation is still triggered, thus enhancing the ISM metallicity, but no effects are visible yet on the temperature. In this scenario, the impact region due to the infall of the "filament" mentioned above would then be a relatively old merger.

Such a scenario appears to be consistent with the various heavy element abundances measured in the Center and in regions Ring 1 and Ring 2, which imply chemical enrichment of the intergalactic gas by both supernovae of type Ia and of type II, and corresponding to different star formation rates.

Therefore, the interpretation of the temperature and metallicity maps derived for the X-ray gas in Abell 85 based on numerical simulations by Fujita et al. (1999) and Bourdin et al. (2004) leads us to suggest that Abell 85 has undergone three mergers, two rather old ones (older than 4 Gyr) from the NW and South, and a more recent one from the NE. Of course, this is only a schematic scenario since it neglects galaxy movements within the cluster, and assumes that galaxies enrich the ISM at their own location, through enhanced star formation and/or ram pressure stripping. Repeated AGN outbursts dredging up material from the center could also produce comparable effects. More detailed modeling obviously requires a better knowledge of the galaxy distribution, in particular of emission line galaxies. We hope to achieve this shortly through multiband imaging allowing us to derive spectroscopic redshifts and $\mathrm{H} \alpha$ imaging to draw the distribution of galaxies with high star formation rates.

Acknowledgements. We thank the referee, Dr. J. C. Kempner, for several interesting suggestions that helped improve our analysis. F.D. and G.B.L.N. acknowledge financial support from the USP/COFECUB and from the PICS France-Brésil from CNRS. G.B.L.N. acknowledges support from FAPESP, CNPq and EARA. This work is based on observations obtained with XMM-Newton, an ESA science mission with instruments and contributions directly funded by ESA Member States and the USA (NASA).

\section{References}

Allen, S. W. 2000, MNRAS, 315, 269

Allen, S. W., Schmidt, R. W., \& Fabian, A. C. 2001, MNRAS, 334, L11

Anders, E., \& Grevesse, N. 1989, Geochim. Cosmochim. Acta, 53, 197

Arabadjis, J. A., \& Bregman, J. N. 1999, ApJ, 510, 806

Bagchi, J., Pislar, V., \& Lima Neto, G. B. 1998, MNRAS, 296, L23

Balucinska-Church, M., \& McCammon, D. 1992, ApJ, 400, 699

Boehringer, H., Voges, W., Fabian, A. C., Edge, A. C., \& Neumann, D. M. 1993, MNRAS, 264, L25

Bourdin, H., Sauvageot, J.-L., Slezak, E., Bijaoui, A., \& Teyssier, R. 2004, A\&A, 414, 429

Bryan, G. L., \& Norman, M. L. 1998, ApJ, 495, 80

Cappellaro, E., \& Turatto, M. 2001, in The influence of binaries on stellar population studies (Brussels, Dordrecht: Kluwer Academic Publisher), 264, 199

Cavaliere, A., \& Fusco-Femiano, R. 1976, A\&A, 49, 137

Demarco, R., Magnard, F., Durret, F., \& Márquez, I. 2003, A\&A, 407, 437

Dickey, J. M., \& Lockman, F. J. 1990, Ann. Rev. Ast. Astr., 28, 215

Donnelly, R. H., Jones, C., Forman, W., Churazov, E., \& Gilfanov, M. 2003 [arXiv: astro-ph/0310145]

Dubinski, J. 1998, ApJ, 502, 141

Dupke, R. A., \& White, R. E. III, 2000a, ApJ, 528, 139

Dupke, R. A., \& White, R. E. III, 2000b, ApJ, 537, 123

Durret, F., Felenbok, P., Lobo, C., \& Slezak, E. 1998a, A\&AS, 129, 281

Durret, F., Forman, W., Gerbal, D., Jones, C., \& Vikhlinin, A. 1998b, A\&A, 335, 41

Durret, F., Lima Neto, G. B., Forman, W., \& Churazov, E. 2003, A\&A, 403, L29

Fabian, A. C., Sanders, J. S., Ettori, S., et al. 2000, MNRAS, 318, L65

Fujita, Y., Takizawa, M., Nagashima, M., \& Enoki, M. 1999, PASJ, 51, L1

Gastaldello, F., \& Molendi, S. 2002, ApJ, 572, 160

De Grandi, S., Ettori, S., Longhetti, M., \& Molendi, S. 2004, A\&A, 419,7

Gerbal, D., Durret, F., Lima Neto, G., \& Lachièze-Rey, M. 1992, A\&A, 253, 77

Grevesse, N., \& Sauval, A. J. 1998, Space Sci. Rev., 85, 161

Hartmann, D., \& Burton, W. B. 1997, Atlas of Galactic Neutral Hydrogen (Cambridge Univ. Press)

Jansen, F., Lumb, D., Altieri, B., et al. 2001, A\&A, 365, L1

Jing, Y. P., \& Suto, Y. 2000, ApJ, 529, L69

Kaastra, J. S., \& Mewe, R. 1993, A\&AS, 97, 443

Kempner, J. C., Sarazin, C. L., \& Ricker, P. M. 2002, ApJ, 579, 236

Komatsu, E., \& Seljak, U. 2001, MNRAS, 327, 1353

Lacey, C., \& Cole, S. 1994, MNRAS, 271, 676

Large, M. I., Cram, L. E., \& Burgess, A. M. 1991, The Observatory, 111,72

Liedahl, D. A., Osterheld, A. L., \& Goldstein, W. H. 1995, ApJ, 438, L115

Lima Neto, G. B., \& Durret, F. 2002, Proc. Conf. Matter and Energy in clusters of galaxies, ASP Conf. Ser., 301, 525, Chung-Li, Taiwan, April 23-27, 2002, ed. S. Bowyer, \& C.-Y. Hwang

Lima Neto, G. B., Pislar, V., Durret, F., Gerbal, D., \& Slezak, E. 1997, A\&A, 327, 81

Lima Neto, G. B., Gerbal, D., \& Márquez, I. 1999, MNRAS, 309, 481 
Lima Neto, G. B., Pislar, V., \& Bagchi, J. 2001, A\&A, 368, 440

Lumb, D. H., Warwick, R. S., Page, M., \& De Luca, A. 2002, A\&A, 389, 93

Markevitch, M., Forman, W. R., Sarazin, C. L., \& Vikhlinin, A. 1998, ApJ, 503, 77

Markevitch, M., Vikhlinin, A., Forman, W. R., \& Sarazin, C. L. 1999, ApJ, 527, 545

Markevitch, M., Ponman, T. J., \& Nulsen, P. E. J. 2000, ApJ, 541, 542

Matteucci, F., \& Gibson, B. K. 1995, A\&A, 304, 11

Moore, B., Quinn, T., Governato, F., Stadel, J., \& Lake, G. 1999, MNRAS, 310, 1147

Nakamura, T., Umeda, H., Iwamoto, K., et al. 1997, ApJ, 555, 880

Navarro, J., Frenk, C. S., \& White, S. D. M. 1997, ApJ, 490, 493

Nomoto, K., Iwamoto, I., Nakasato, N., et al. 1997, Nuclear Phys. A, $621,467 \mathrm{c}$

Oegerle, W. R., \& Hill, J. M. 2001, AJ, 122, 2858

Pislar, V., Durret, F., Gerbal, D., Lima Neto, G. B., \& Slezak, E. 1997, A\&A, 322, 53
Pepino, A., Matteucci, F., Borgani, S., \& Biviano, A. 2002, New Astron., 7, 227

Pointecouteau, E., Arnaud, M., Kaastra, J., \& de Plaa, J. 2004, A\&A, 423, 33

Rasia, E., Tormen, G., \& Moscardini, L. 2004, MNRAS, 351, 237

Sanders, J. S., Fabian, A. C., Allen, S. W., \& Schmidt, R. W. 2004, MNRAS, 349, 952

Sérsic, J. L. 1968, Atlas de Galaxias Australes, Cordoba, Argentina Slee, O. B., Roy, A. L., Murgia, M., Andernach, H., \& Ehle, M. 2001, AJ, 122, 1172

Slezak, E., Durret, F., Guibert, J., \& Lobo, C. 1998, A\&AS, 128, 67

Tamura, T., Kaastra, J. S., den Herder, J. W. A., Bleeker, J. A. M., \& Peterson, J. R. 2004, A\&A, 420, 135

Wilms, J., Allen, A., \& McCray, R. 2000, ApJ, 542, 914

White, S. D. M., Navarro, J. F., Evrard, A. E., \& Frenk, C. S. 1993, Nature, 366, 429 